\title{
ON CLUSTERING IN CENTRAL CONFIGURATIONS
}

\author{
GREGORY BUCK
}

(Communicated by Kenneth R. Meyer)

\begin{abstract}
Central configurations lead to special solutions of the $n$-body problem. In this paper we present a geometric condition that all central configurations must satisfy: a central configuration cannot have too much 'clustering'they are bounded away from the diagonal in configuration space. An explicit bound is given.
\end{abstract}

\section{INTRODUCTION}

The Newtonian $n$-body problem is intractable for $n \geq 3$. One approach is then to look for particular solutions. In this spirit we consider central configurations. Central configurations are initial arrangements of the bodies that lead to special solutions of the $n$-body problem. Roughly speaking, they are solutions that remain self-similar for all time-that is, the ratios of the mutual distances between the bodies remain constant. There is extensive literature concerning these solutions. For a comprehensive introduction the reader should see [Saari] and the references therein. Recent work includes [Meyer-Schmidt], [Moekel], [Hall], and [Simo].

Here a general property of all central configurations is presented. A concept of clustering is defined and it is shown that in a central configuration the masses cannot be too tightly clustered. This extends an important result of M. Shub's (see below and [Shub]). The proof is geometric in nature.

We shall need some definitions and terminology. These are standard to Celestial Mechanics discussions. (See [Saari])

Definition 1. Notation

A configuration $X=\left\{x_{1}, \ldots, x_{n}, m_{1}, \ldots, m_{n}\right\}$ of $n$ bodies is a choice of positions $x_{1}, \ldots, x_{n} \in \mathbf{R}^{3}$ and masses $m_{1}, \ldots, m_{n} \in \mathbf{R}$.

The potential of a configuration, denoted $U(X)$, is defined as

$$
U(X)=\sum_{i<j} \frac{m_{i} m_{j}}{\left|x_{i}-x_{j}\right|} .
$$

Received by the editors November 7, 1988.

1980 Mathematics Subject Classification (1985 Revision). Primary 70F10.

Key words and phrases. $n$-body problem, central configurations, relative equilibria.

The author's research was partially supported by the National Science Foundation. 
The moment of inertia of a configuration, denoted $I(X)$, is defined as

$$
I(X)=\frac{1}{2} \sum_{i=0} m_{i}\left|x_{i}\right|^{2}
$$

The force on the $i$ th body of a configuration, denoted $F\left(x_{i}\right)$ or $F_{i}$, is

$$
F_{i}=\sum_{j \neq i} \frac{m_{i} m_{j}\left(x_{i}-x_{j}\right)}{\left|x_{i}-x_{j}\right|^{3}} .
$$

The acceleration of the $i$ th body, denoted $A\left(x_{i}\right)$ or $A_{i}$, is

$$
A_{i}=\sum_{j \neq i} \frac{m_{j}\left(x_{i}-x_{j}\right)}{\left|x_{i}-x_{j}\right|^{3}} .
$$

The equations of motion, or Newton's equations, are

$$
m_{i} \ddot{x}_{i}=F\left(x_{i}\right), \quad i=1, \ldots, n
$$

(where $\cdot$ represents the derivative with respect to time).

A central configuration is a configuration such that if the initial velocities $\dot{x}_{1}(0)=\cdots=\dot{x}_{n}(0)=0$, then the solution of the equations of motion is of the form $x_{i}(t)=\varphi(t) x_{i}$ for all $i$, where

$$
\varphi\left[0, t^{*}\right] \rightarrow[0,1] \quad \text { with } \varphi(0)=1 \text { and } \varphi\left(t^{*}\right)=0 .
$$

That is, starting at rest the configuration collapses homothetically.

A necessary and sufficient condition for a configuration to be central is:

$$
A_{i}=k x_{i}, \quad \text { for all } i \text {, where } k \text { is a constant independent of } i \text {. }
$$

We shall sometimes refer to (1) as the central configuration equations, and in practice will use (1) as our definition. See [Saari] for more on the definitions of central configuration.

As it stands under the definition, the class of central configurations is larger than it need be. We need not distinguish between configurations that differ only by translation or rotation or a combination thereof. Additionally, we can call configurations equivalent if they differ only by scale-this is sensible both by the definition and by the equations (1). If two central configurations differ only by scale, the larger would collapse through the smaller if begun at rest. In terms of the central configuration equations, configurations that differ by scale differ in the constant $k$ in equations (1). The standard procedure is to choose a representative of the equivalence classes, say by setting $I=1$. An alternative is a choice for $k$ in the central configuration equations. Another alternative, employed below, is to set a particular mutual distance $\left|x_{i}-x_{j}\right|=1$.

It is known that central configurations are critical points of the potential restricted to the sphere where the moment of inertia is equal to a constant. In [Shub] M. Shub showed that under these conditions the potential does not have any singularities in a neighborhood of the diagonal, that is, where $x_{i}=x_{j}$, 
$i \neq j$. This implies that central configurations do not limit onto the diagonal. In the following we find an explicit neighborhood of the diagonal that has no central configurations, giving an alternative proof of Shub's result, as well as an explicit bound for the size of the neighborhood. The bound depends on the masses of the bodies, the mutual distances between the bodies, and the number of bodies in the configuration. This gives a bound on "clustering" in central configurations-a configuration such as that in Figure 1 cannot be central. In the following the case of planar configurations is discussed, the proof goes over to spatial configurations with hardly any changes, resulting in the same estimates.

We note that in [Schmidt] D. Schmidt gives inequalities that must be satisfied in the cases of four and five bodies. These provide some bounds on clustering in these cases.

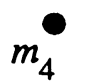

$$
m_{5}^{0}
$$
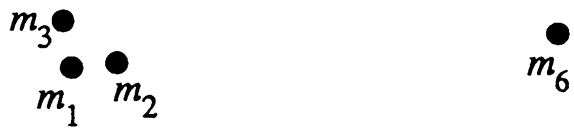

FIGURE 1. Let $m_{1}=m_{2}=\cdots=m_{6}$. Then from the results in this paper (Theorems 1,2) this configuration cannot be central, because $m_{1}, m_{2}, m_{3}$, are too clustered.

\section{THE THEOREM FOR EQUAL MASSES}

We begin with the case of $n$ bodies of equal mass, and show later how masses enter the calculations.

First normalize the configuration so that the center of mass is at the origin, and the distance from the center of mass to the mass furthest from the origin is 1 .

Let $\left\{r_{i j}\right\}$ be the set of mutual distances of the bodies in an arbitrary central configuration, arranged in increasing order. Let $\rho_{1}, \rho_{2}$ be consecutive entries in the list $\left\{r_{i j}\right\}$.

In a central configuration $\left|A_{i}\right| /\left|x_{i}\right|=\left|A_{j}\right| /\left|x_{j}\right| \forall_{i, j}$, where $A_{i}$ is the acceleration vector associated with the $i$ th mass, $x_{i} \in \mathbf{R}^{2}$ the position of the $i$ th mass. We find the bound on clustering by finding a lower bound for a particular 
$\left|A_{a}\right| /\left|x_{a}\right|$, an upper bound for a particular $\left|A_{b}\right| /\left|x_{b}\right|$, then computing a value of a function $g\left(\rho_{1}, \rho_{2}\right)$ such that $\left|A_{b}\right| /\left|x_{b}\right|<\left|A_{a}\right| /\left|x_{a}\right|$.

\section{A LOWER BOUND FOR $\left|A_{a}\right| /\left|x_{a}\right|$}

Choose $x_{a}, x_{k}$ such that $\left|x_{a}-x_{k}\right|=\rho_{1}$.

Define the cluster about $x_{a}$ as the unique set of bodies contained in the intersection of $B\left(x_{a}, \rho_{1}\right)$ and $B\left(x_{k}, \rho_{1}\right)$, where $B\left(x_{i}, \rho_{j}\right)$ is the closed disk of radius $\rho_{j}$ centered at $x_{i}$.

Proposition 1. Any bodies not in the cluster about $x_{a}$ are at least $\rho_{2}-\rho_{1}$ from $x_{a}$.

Proof. Any body not in the cluster is at least $\rho_{2}$ from either $x_{k}$ or $x_{a}$. If the body is $\rho_{2}$ from $x_{k}$, it is at least $\rho_{2}-\rho_{1}$ from $x_{a}$ (see Figure 2).

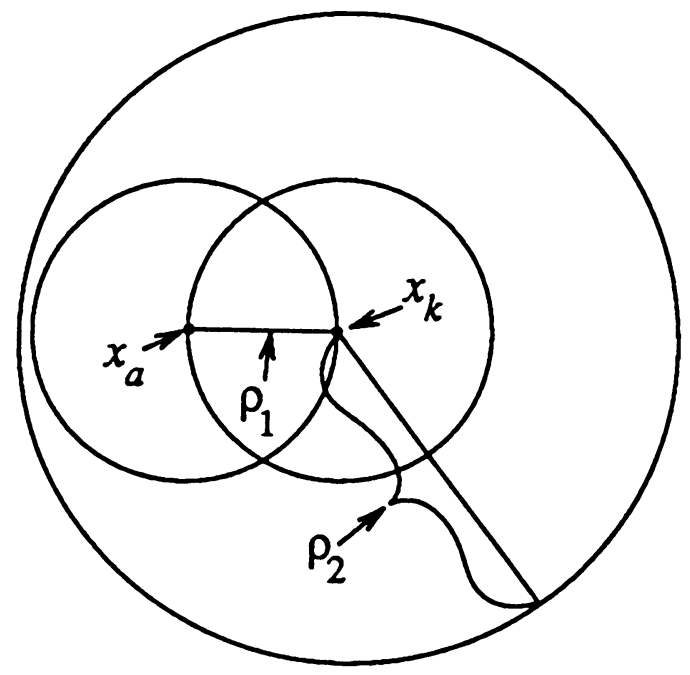

FIGURE 2.

Definition. Let $A_{a}^{*}$ be the component of the acceleration vector $A_{a}$ resulting from the members of the cluster about $x_{a}$.

Proposition 2. Let the cardinality of the cluster about $x_{a}$ be $m$. Then $\left|A_{a}^{*}\right| \geq$ $m / 2\left(\rho_{1}\right)^{2}$.

Proof. A body in the cluster about $x_{a}$ is contained in $B\left(x_{a}, \rho_{1}\right) \cap B\left(x_{k}, \rho_{1}\right)$. The minimum possible force exerted on $x_{a}$ in the direction of $x_{k}$ by the body is achieved on the boundary of $B\left(x_{a}, \rho_{1}\right) \cap B\left(x_{k}, \rho_{1}\right)$. To see this consider the rays from $x_{a}$ into the region. The force strictly decreases along these rays. The boundary of the region has two parts: $s_{1} \subset B\left(x_{a}, \rho_{1}\right), s_{2} \subset B\left(x_{k}, \rho_{1}\right)$ (see Figure 3). 


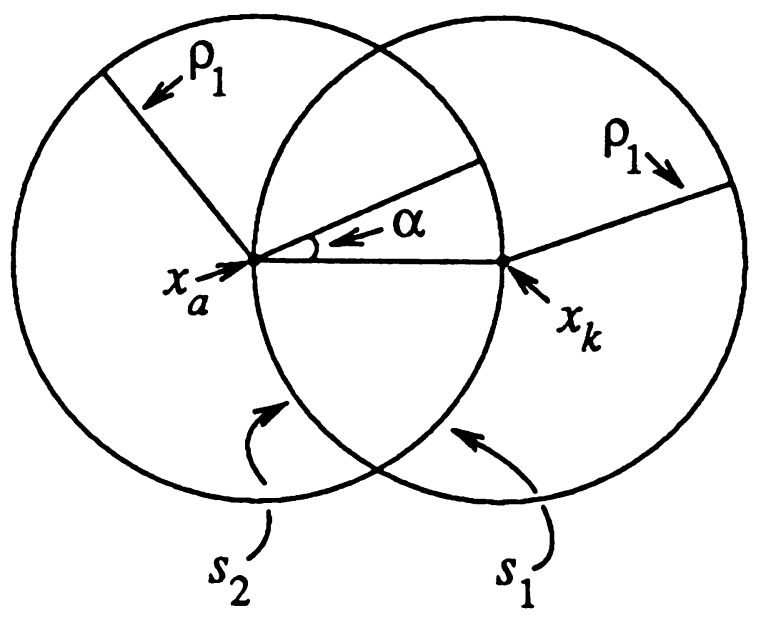

FIGURE 3.

The minimum along $s_{1}$ is seen to be at $s_{1} \cap s_{2}$, since the distance from $x_{a}$ to $s_{1}$ is a constant $\rho_{1}$; so we seek to minimize $\cos \alpha$, when $\alpha$ is the angle between the ray from $x_{a}$ to $x_{k}$ and the ray from $x_{a}$ to the body. (See Figure 3.)

For the minimum along $s_{2}$, we consult the diagram (Figure 4) (let $\left.\rho_{1}=1\right)$ :

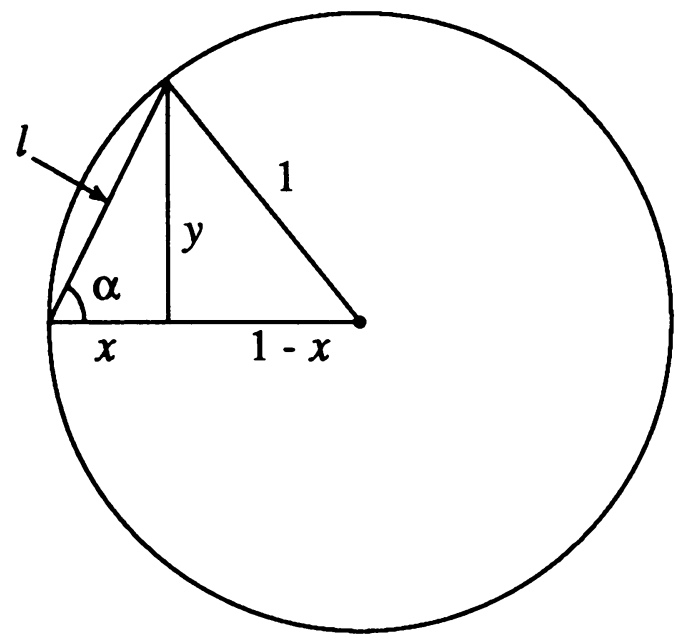

Figure 4.

The component of the force we are interested in is $(\cos \alpha) / l^{2}$. Now $l^{2}=$ $x^{2}+y^{2}, y=\sqrt{2 x-x^{2}}$ so $l=\sqrt{2 x} \cos \alpha=x / l$, so $(\cos \alpha) / l^{2}=1 / 2 \sqrt{2 x}$, which decreases strictly as $x$ increases, so the minimum takes place for $\max \{x\}$ for $x \in s_{2}$, which is $s_{2} \cap s_{1}$, where $l=\rho_{1}$ and $\alpha=\pi / 3$. This completes the procf of the proposition, since $\left|A_{a}^{*}\right| \geq\left|A_{a}^{*} \cdot\left[\left(x_{k}-x_{a}\right) /\left|x_{k}-x_{a}\right|\right]\right|$. 
Proposition 3. $\left|A_{a}\right| \geq m / 2\left(\rho_{1}\right)^{2}-(n-m) /\left(\rho_{2}-\rho_{1}\right)^{2}$, where $n$ is the number of bodies in the configuration, and $m$ is the number of bodies in the cluster about $x_{a}$.

Proof. We have from Proposition 2 that $\left|A_{a}^{*}\right| \geq m / 2\left(\rho_{1}\right)^{2}$. So the worst case is that the remaining masses, which are at least $\rho_{2}-\rho_{1}$ from $x_{a}$, lie $\rho_{2}-\rho_{1}$ from $x_{a}$ along the line joining $x_{a}$ and the center of mass. The estimate follows.

Now $\left|x_{a}\right| \leq 1$ from the normalization, so

$$
\frac{\left|A_{a}\right|}{\left|x_{a}\right|} \geq \frac{m}{2\left(\rho_{1}\right)^{2}}-\frac{n-m}{\left(\rho_{2}-\rho_{1}\right)^{2}} \text {. }
$$

\section{AN UPPER BOUND FOR $\left|A_{b}\right| /\left|x_{b}\right|$}

First we choose $x_{b}$. Consider $x_{j}$, a mass maximum distance $(=1)$ from the center of mass. If $B\left(x_{j}, \rho_{1}\right)$ contains only $x_{j}$, then let $x_{b}=x_{j}$. Otherwise:

Consider the convex hull of the masses contained in $B\left(x_{j}, \rho_{1}\right)$. Let $r_{j}$ be the ray representing the shortest distance from the center of mass to the convex hull of the masses in $B\left(x_{j}, \rho_{1}\right)$. There are two cases here, both employ:

Proposition 4. $A_{b}^{*}$, where $x_{b}$ is a vertex of a convex hull of masses (the * here signifies the acceleration resulting from the bodies in the convex hull), points toward the interior of the convex hull.

Proof. Elementary. Each component vector is inside the convex hull (see Figure 5).

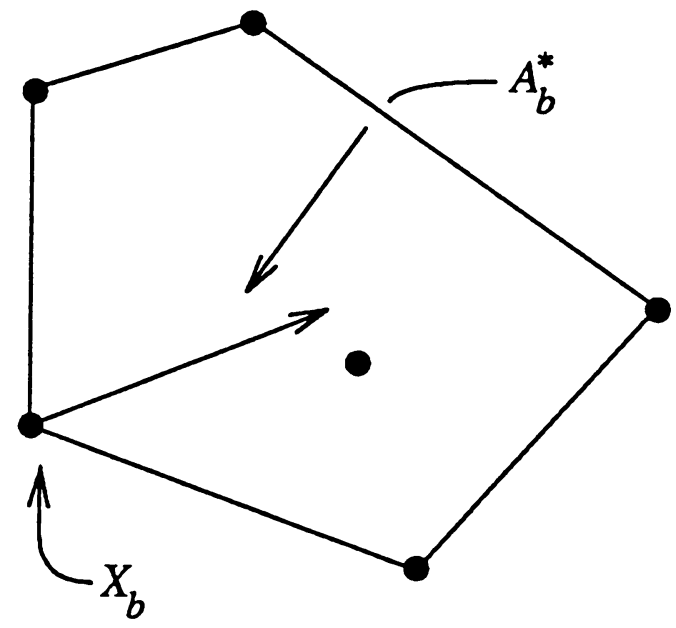

FIGURE 5. 
The two cases are:

1. $r_{j}$ meets the convex hull at a vertex. Then the convex hull lies beyond the perpendicular to the endpoint of $r_{j}$, and we let $x_{b}$ be the body at the vertex where $r_{j}$ meets the hull. So from Proposition $4, A_{b}^{*}$ is as in Figure 6.

2. $r_{j}$ meets the convex hull on a side. But then $r_{j}$ is perpendicular to the side, since a unique intersection between a line and a circle must be perpendicular to the ray joining the intersection and the center. So $A_{b}^{*}$, where $x_{b}$ is either vertex on the end of the side, lies above the perpendicular to $r_{j}$. (See Figure 6.)

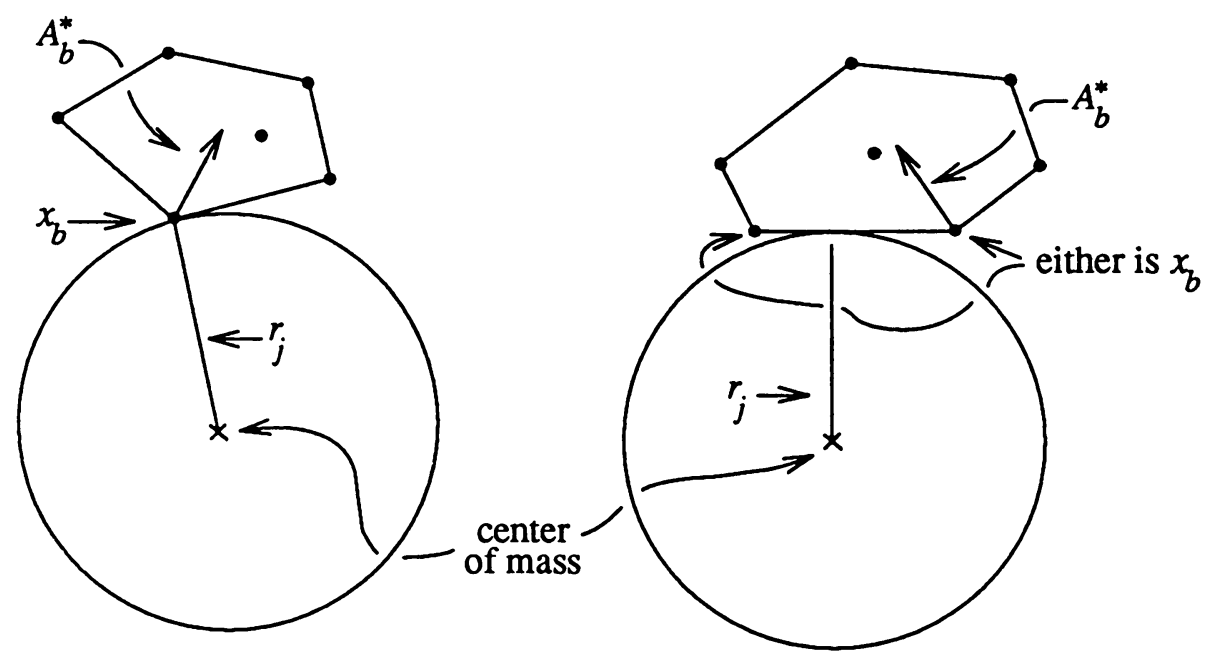

Figure 6.

In either case we have:

Proposition 5. $\left|A_{b}\right| \leq(n-1) /\left(\rho_{2}-\rho_{1}\right)^{2}$.

Proof. The vector $A_{b}$ must 'point at' the center of mass, since we have assumed the configuration is central. So the best case is that $A_{b}^{*}=0$ and the remaining masses are along the line joining $x_{b}$ and the center of the mass-by choice of $\rho_{1}$ and $\rho_{2}$ at least $\left(\rho_{2}-\rho_{1}\right)$ away.

\section{Proposition 6.}

$$
\frac{\left|A_{b}\right|}{\left|x_{b}\right|} \leq \frac{(n-1) /\left(\rho_{2}-\rho_{1}\right)^{2}}{1-\rho_{1}}
$$

Proof. Proposition 5 and $\left|x_{b}\right| \geq 1-\rho_{1}$ by choice of $x_{b}$. 


\section{COMPUTATION OF BOUND}

We have that

$$
\left|A_{a}\right| /\left|x_{a}\right| \geq m / 2\left(\rho_{1}\right)^{2}-(n-m) /\left(\rho_{2}-\rho_{1}\right)^{2}
$$

and

$$
\frac{\left|A_{b}\right|}{\left|x_{b}\right|} \leq \frac{(n-1) /\left(\rho_{2}-\rho_{1}\right)^{2}}{1-\rho_{1}}
$$

So we need to find when

$$
\frac{m}{2\left(\rho_{1}\right)^{2}}-\frac{n-m}{\left(\rho_{2}-\rho_{1}\right)^{2}}>\frac{(n-1) /\left(\rho_{2}-\rho_{1}\right)^{2}}{1-\rho_{1}}
$$

for some choice of $\rho_{1}, \rho_{2}$ given $n, m$.

so

$$
\left(1-\rho_{1}\right)\left(\rho_{2}-\rho_{1}\right)^{2}\left[\frac{m}{2\left(\rho_{1}\right)^{2}}-\frac{(n-m)}{\left(\rho_{2}-\rho_{1}\right)^{2}}\right]>n-1
$$

so

$$
\left(1-\rho_{1}\right)\left(\rho_{2}-\rho_{1}\right)^{2}\left[\frac{\left(\rho_{2}-\rho_{1}\right)^{2} m-2\left(\rho_{1}\right)^{2}(n-m)}{2\left(\rho_{1}\right)^{2}\left(\rho_{2}-\rho_{1}\right)^{2}}\right]>n-1
$$

so

$$
\frac{\left(1-\rho_{1}\right)}{2\left(\rho_{1}\right)^{2}}\left[\left(\rho_{2}-\rho_{1}\right)^{2} m-2\left(\rho_{1}\right)^{2}(n-m)\right]>n-1
$$

so

$$
\frac{\left(1-\rho_{1}\right)}{2\left(\rho_{1}\right)^{2}}\left(\rho_{2}-\rho_{1}\right)^{2} m-\left(1-\rho_{1}\right)(n-m)>n-1
$$

$$
\frac{\left(1-\rho_{1}\right)}{2\left(\rho_{1}\right)^{2}}\left(\rho_{2}-\rho_{1}\right)^{2} m>(n-1)+\left(1-\rho_{1}\right)(n-m) .
$$

We know $n>m$, so we make the right-hand side as large as possible by dropping the $\rho_{1}$ term, so if

$$
\frac{1-\rho_{1}}{2\left(\rho_{1}\right)^{2}}\left(\rho_{2}-\rho_{1}\right)^{2}>\frac{2 n-m-1}{m}
$$

we have that $\left|A_{a}\right| /\left|x_{a}\right|>\left|A_{b}\right| /\left|x_{b}\right|$ and the configuration cannot be central. This gives

Theorem 1. Let $X$ be a configuration of $n$ equal masses, normalized so that the center of mass is at the origin and $\left|x_{l}\right|=1$, where $x_{l}$ is the mass furthest from the center of mass. Let $\rho_{1}, \rho_{2}$ be consecutive entries in $\left\{r_{i_{j}}\right\}$, the list of mutual distances of $X$ arranged in increasing order. Let $m$ be the number of masses in the $\rho_{1}$ cluster about some $x_{i}(m \geq 1$ always-see below). Then if

$$
\frac{1-\rho_{1}}{2\left(\rho_{1}\right)^{2}}\left(\rho_{2}-\rho_{1}\right)^{2}>\frac{2 n-m-1}{m}
$$

the configuration is not central. 
The fact that $\left(\left(1-\rho_{1}\right) / 2\left(\rho_{1}\right)^{2}\right)\left(\rho_{2}-\rho_{1}\right)^{2} \rightarrow \infty$ as $\rho_{1} \rightarrow 0$ gives that we have a neighborhood of the diagonal without central configurations for any given $n$, since if we fix $n$ then some $\rho_{i}$ is at least $1 / n$.

From the definition of cluster we have that $m \geq 1$. So if we assume $m=1$ the bound simplifies to:

$$
\frac{1-\rho_{1}}{2\left(\rho_{1}\right)^{2}}\left(\rho_{2}-\rho_{1}\right)^{2}>2 n-2
$$

\section{HOW MASSES ENTER THE COMPUTATION}

We let $m_{1}$ be the smallest mass in $X, m_{2}$ the largest, and consider the worst case for the estimates. Then

$$
\frac{\left|A_{a}\right|}{\left|x_{a}\right|} \geq \frac{\left(m_{1}\right)^{2} m}{2\left(\rho_{1}\right)^{2}}-\frac{\left(m_{2}\right)^{2}(n-m)}{\left(\rho_{2}-\rho_{1}\right)^{2}} .
$$

This is where the smallest masses are in the cluster about $x_{a}$, the largest outside the cluster. Similarly,

$$
\frac{\left|A_{b}\right|}{\left|x_{b}\right|} \leq \frac{\left(\left(m_{2}\right)^{2}(n-1)\right) /\left(\rho_{2}-\rho_{1}\right)^{2}}{\left(1-\rho_{1}\right)}
$$

so the computation begins with

$$
\frac{\left(m_{1}\right)^{2} m}{2\left(\rho_{1}\right)^{2}}-\frac{\left(m_{2}\right)^{2}(n-m)}{\left(\rho_{2}-\rho_{1}\right)^{2}}>\frac{\left(\left(m_{2}\right)^{2}(n-1)\right) /\left(\rho_{2}-\rho_{1}\right)^{2}}{1-\rho_{1}} .
$$

Continuing as before gives

$$
\frac{\left(1-\rho_{1}\right)}{2\left(\rho_{1}\right)^{2}}\left[m\left(m_{1}\right)^{2}\left(\rho_{2}-\rho_{1}\right)^{2}-\left(m_{2}\right)^{2} 2\left(\rho_{1}\right)^{2}(n-m)\right]>\left(m_{2}\right)^{2}(n-1),
$$

so we get

$$
\frac{\left(1-\rho_{1}\right)}{2\left(m_{2}\right)^{2}\left(\rho_{1}\right)^{2}}\left(m\left(m_{1}\right)^{2}\left(\rho_{2}-\rho_{1}\right)^{2}\right)-\left(1-\rho_{1}\right)(n-m)>n-1
$$

and so if

$$
\frac{\left(1-\rho_{1}\right)\left(\rho_{2}-\rho_{1}\right)^{2}}{2\left(\rho_{1}\right)^{2}} \frac{\left(m_{1}\right)^{2}}{\left(m_{2}\right)^{2}}>\frac{2 n-m-1}{m}
$$

the configuration is not central. This gives:

Theorem 2. Let $X$ be a configuration with masses $m_{1}, \ldots, m_{n}$. Let $m_{2}=$ $\max \left\{m_{i}\right\}$, let $m_{1}=\min \left\{m_{i}\right\}$. Otherwise assume the same hypotheses as Theorem 1 . Then if

$$
\frac{\left(1-\rho_{1}\right)\left(\rho_{2}-\rho_{1}\right)^{2}}{2\left(\rho_{1}\right)^{2}}\left(\frac{m_{1}}{m_{2}}\right)^{2}>\frac{2 n-m-1}{m}
$$

the configuration is not central. 
Spatial configurations. As mentioned in the introduction, the arguments go over to spatial configurations with hardly any changes, and give the same estimates. The lower bound on $\left|A_{a}\right| /\left|x_{a}\right|$ employs a region between intersection spheres. For the upper bound on $\left|A_{b}\right| /\left|x_{b}\right|$, one shows that $A_{b}^{*}$ is restricted to a half space (instead of a half plane) some distance from the center of mass.

\section{REFERENCES}

[Hall] G. R. Hall, Central configurations of the $1+N$ body problem, preprint.

[Meyer-Schmidt] K. Meyer and D. Schmidt, Bifurcation of relative equilibria in the four and five body problem, preprint.

[Moekel] R. Moekel, Relative equilibria of the four-body problem, Ergodic Theory and Dynamical Systems Vol. 5, part 3, (1985), 417-435.

[Saari] D. Saari, On the role and properties of $n$-body central configurations, Celestial Mech. 21 (1980).

[Schmidt] D. Schmidt, Central configurations in $\mathbf{R}^{2}$ and $\mathbf{R}^{3}$, preprint.

[Shub] M. Shub, Appendix to Smale's paper: "Diagonals and relative equilibria," ManifoldsAmsterdam 1970, Lecture Notes in Math., Vol. 197, Springer, Berlin, 1971, 199-201.

[Simo] C. Simo, Relative equilibrium solutions in the four body problem, Celest. Mech. 18 (1977), 165-184.

[Smale] S. Smale, Problems on the nature of relative equilibria in celestial mechanics, ManifoldsAmsterdam 1970, Lecture Notes in Math, Vol. 197, Springer, Berlin, 1971, 194-198.

Department of Mathematics, Boston University, Boston, Massachusetts 02215

Current address: Department of Mathematics, Tufts University, Medford, Massachusetts 02155 